\title{
Design of Humidity-Controlled Exhaust Fan with Lighting Function
}

\author{
K.F. Yarn* \\ Department of Aircraft Maintenance, Far East University, Taiwan 74448, ROC \\ ymo86@yahoo.com.tw \\ *corresponding author
}

\begin{abstract}
The production implemented in this article is mainly composed of humidity sensing control, A/D conversion, fan, refrigeration chip, 89C51, LED, LCD panel and other circuit combinations. When the humidity reaches the set value, the system will automatically start the de-humidification effect. Measure the numerical value of the humidity of the acrylic box to determine whether the current humidity status of the acrylic box reaches the set numerical humidity and start the device, convert the digital signal through the AD0804 analog signal, and transfer the signal to the 89C51 chip, and send it to LCD display and start the application function of the fan and cooling chip combined device to the LCD display to reduce the humidity value of the acrylic box and achieve the de-humidification effect studied by this implementation.
\end{abstract}

Keywords: Humidity Sensing, Cooling chip, Energy Saving, Fan

\section{Introduction}

Taiwan has a subtropical or subtropical climate, in which the north is a subtropical monsoon climate zone, and the south is a tropical monsoon climate zone. On the other hand, Taiwan is surrounded by the sea, which is affected by warm and humid air currents and ocean currents. Therefore, it has an oceanic climate and because it is close to the mainland, and the weather system mostly moves from west to east, Taiwan is inevitably subject to continental impact of climate. In addition, although the area of Taiwan is small, the central and eastern regions are all alpine terrains. Mountains, hills and terraces with heights above 500 meters account for about $46 \%$ of the total area. The central mountain range and snow-capped mountain range run from north to south. The Yushan and Yushan Mountains are all mountainous areas with a height of more than 3,000 meters. In the west, there are still Alishan Mountains with a height of between one and two thousand meters. These have a significant impact on the "Taiwan climate". The mountain and flat land, the south and the north, the west and the east show very different climatic characteristics of rainfall patterns and temperature changes. [1-2]

"Tropical island climate", the high temperature and humidity environment is a good environment for bacteria growth. The common relative humidity is as high as $80-90 \%$, which is much higher than the normal relative humidity $(60 \%)$; in addition, the ventilation of the building is not easy. It is easier to grow bacteria during the exchange of seasons. Because of "high humidity", "low temperature" and "slow water evaporation". The reason is the rapid growth of bacteria.

Therefore, moisture is the most important factor. When the microbial mold multiplies, it will cause many harms and property losses. If the wall on the wall cancer is found to turn from white to green or black, it 
means that the house is occupied by bacteria and mold, and it begins to affect the home environment and physical and mental health. Health produces invisible damages and diseases. Mould floats into the home environment with the air, causing continuous reproduction and breeding, eroding the entire home and surrounding environment. However, in Taiwan's climate, in a hot and humid environment, mold spores floating in the air will fall on organic food and material products, such as dry food, wood, fiber, leather, walls, and so on. Attaching to these foods and supplies causes mold to germinate, grow hyphae, spread infestation and disease. As a result, the materials of building materials, food, clothing, furniture, etc. are deteriorated, deteriorated, decayed, etc., and lose their original functions. It is called "mildew".

Because all commercially available dehumidifiers use refrigerant as the main means of dehumidification, and refrigerant is a substance that easily absorbs heat and becomes a gas, but also easily becomes a liquid when it releases heat. Regardless of whether environmentally friendly refrigerants or traditional refrigerants, there are fluorides or chlorides that can harm the human body or damage the environment. Among them, the refrigerant containing chloride is the main killer that destroys the atmosphere. It will destroy the earth's ozone layer, making the earth's ozone layer facing a crisis of holes, which in turn causes ultraviolet rays to directly penetrate the atmosphere and damage people's skin, causing people to suffer from various diseases. Skin diseases and skin lesions can even evolve into skin cancer. The main ozone destroyer in the refrigerant, CFC12, may be replaced by HFC-134a, but the chemical engineer said that HFC-134a is more difficult to manufacture, the price is more expensive than $\mathrm{CFC}-12$, and more frequent replacement than $\mathrm{CFC}-12$. Alternatives to CFC-11, CFC-12 and CFC-113 still need long-term research and development, because many alternatives are industrially. [3-4]

The properties are inferior to that of chlorofluorocarbons, and they are less durable, and even more equipment must be designed to use them. These substitutes are easy to decompose under low pressure, but they are less threatening to the ozone layer. However, human exposure to these substitutes will be potentially dangerous or cause other environmental problems. In addition, the environmentally friendly refrigerant HCFC is relatively unstable and has a shorter life span in the atmosphere, and is easily decomposed before the environmentally friendly refrigerant HCFC reaches the ozone layer. However, in animal experiments, it was found that HCFC has severe hepatotoxicity to guinea pigs, and caused slight hepatic necrosis in mice and dogs to induce abnormal behaviors and liver tumors, and studies have pointed out that long-term exposure to HCFC environment will cause benign tumors. Find. Therefore, although the ozone content of the ozone layer is extremely small, it also has a strong absorption.

The function of receiving ultraviolet rays can absorb the harmful part (UVB) of the ultraviolet rays of the sun. Therefore, to protect the destruction of the ozone layer, it can effectively block the invasion of ultraviolet rays from the sun, so that human beings and various life on the earth can exist, multiply and develop. In view of this, the use of refrigeration chips to replace refrigerants in the implementation of this article has become more important and urgent for the protection of the ozone layer and human health. [5-6]

\section{Motivation for Production}

This article implements the "Humidity-Controlled Exhaust Fan with Lighting Function", which mainly combines refrigeration chips, electronic solid-state lighting and humidity sensing components. Through this topic, the humidity controllable cabinet can be realized. Not only can the knowledge learned in the school be combined with theories, but also can be applied to daily life applications, and can be cultivated for future scientific and technological fields and applications.

This article implements the production because every family needs a kitchen. The cabinet under the flow table is equipped with drain pipes, and the closed space under the flow table causes the humidity in the cabinet to be heavy, and it is easier to breed mold. The role of mold makes the material deteriorate, deteriorate, 
decompose, and corrosion, collapse and other functions; not only boring, unsightly, but also difficult to eradicate on the surface, causing microbial disasters. Kitchen utensils are often put in the cupboard. For example: rice, soup spoons, pots, plates, knives and other items are made by our people to improve the moisture in the cabinet.

In general household kitchens, the humidity in the cabinets is heavier. Since most of the dehumidifiers currently on the market use refrigerants as the medium, the fact that the body is overheated due to the control interface problems in use is often heard, so the fact that the dehumidifiers This report of fire and burning so exclude the dehumidifier. In addition, due to the refrigerant used can cause damage to the environment. Therefore, we are taking precautionary measures to protect the environment nowadays. Therefore, the disadvantage of dehumidifiers is that they are large in size and occupy space. However, operating in a closed space is more likely to cause high temperature and overheating of the body, causing electrical products to ignite, and refrigerant leakage that causes environmental damage, and so on.

In addition, considering that there is insufficient light in the cabinet, it will be inconvenient to find things or repair water pipes. Therefore, in the implementation of this article, we will combine environmentally friendly and energy-saving white LEDs as auxiliary lighting equipment. This will not only provide considerable convenience. It can also achieve the function of lighting and low power consumption and power saving. This article implements the use of a cooling chip and a humidity sensing device combined with a fan to achieve the dehumidification effect. The humidity sensor and the fan are operated to draw the internal moisture out of the outer head and be cooled by the cooling chip and become water droplets. The initial idea is the functioning principle of the dehumidifier, which reminds me of the cooling chip of the internal device. Therefore, the implementation of this article envisages this solution to discharge the moisture in the cabinet to reduce the internal moisture and to prevent the food stored in the cabinet from being perishable and moldy. This implementation can achieve the purpose of dehumidification.

The fan must be combined with the cooling chip to dehumidify. The cooling chip can be cooled or heated by only inputting electric current, and it can respond to the concept of environmental protection without using a refrigerant. However, using the extracted hot air to cool and exhaust, on the one hand, it does not occupy space but can also achieve power saving and convenience. Therefore, using the combination of a fan and a cooling chip for dehumidification can not only reduce the use of refrigerants, save energy and save electricity, but also achieve the effect of not occupying space. It can make it convenient for people to use the device.

The implementation of this article hopes to use the combination of humidity sensing and fan to achieve effective applications. It can also set the humidity setting to the required value and activate the device to remove moisture in the cabinet. However, the white light LED lighting function is installed so that the place where things are placed can be clearly seen without having to turn on the household electric light to consume electricity, and the white light LED light can achieve low power consumption and save electricity and have better lighting effects.

\section{Experimental}

Refrigeration wafers are semiconductor elements that can be freely cooled, heated, and temperature controlled by a DC power supply. The temperature difference between the two sides of the refrigerating chip is affected by the magnitude of the current, and the greater the current, the greater the temperature difference. The main materials of refrigeration chips: antimony, tellurium, bismuth, and selenium are all rare elements.

Refrigeration chip principle: A piece of N-type semiconductor and a piece of P-type semiconductor material are connected to form a galvanic pair. After the DC current is connected in this circuit, energy transfer can occur. The current flows from the N-type element to the P-type element to absorb heat and become the cold junction. The current flows from the P-type element to the joint of the N-type element to 
release heat and become the hot end. The amount of heat absorbed and released is determined by the magnitude of the current. Figure 1 is a structural diagram of a refrigerating wafer.

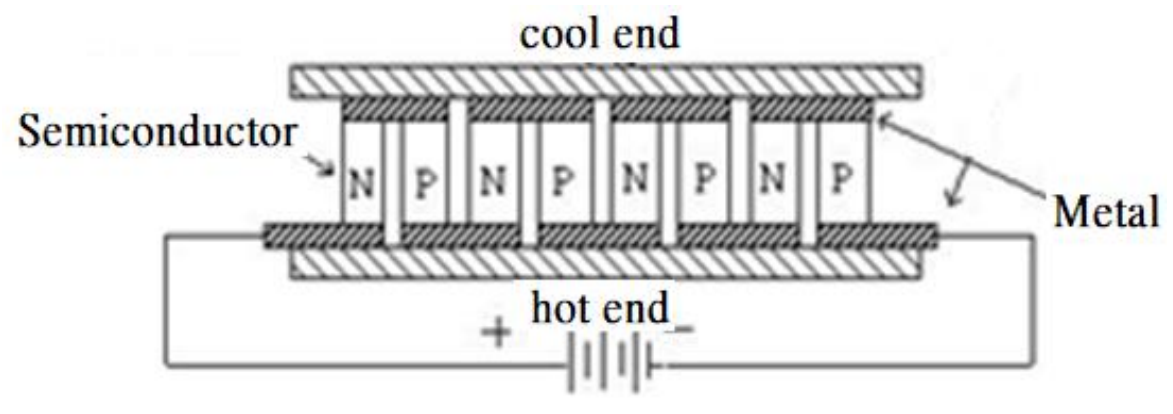

Figure 1. Refrigeration chip structure diagram

Generally, the maximum temperature difference of products on the market is $62^{\circ} \mathrm{C}$. Assuming that the temperature of the hot end is $27^{\circ} \mathrm{C}$, and the temperature difference between the cold end and the hot end is $62^{\circ} \mathrm{C}$, the coldest cooling chip can reach $-35^{\circ} \mathrm{C}$. Advantages of refrigeration wafers compared with traditional refrigeration compressors, refrigeration wafers have advantages but also disadvantages. It is small in size, noise-free, and does not use cold coal, so it is environmentally friendly and has a long service life. There is no direction limit, and it can be used upside down or sideways. The cost is high, but there is almost no need for maintenance in the future. The refrigerating chip can be used in series or in parallel, which can increase the flexibility of the refrigerated chip and make it suitable for more occasions. The disadvantage of refrigerating chips is low energy conversion efficiency. Therefore, refrigeration chips cannot be used in large air conditioners or large refrigerators.

Refrigeration chips can be used in daily necessities, aerospace industry, medical bioassay, military and civilian industries, etc. The most common uses include computer CPU cooling, dehumidification boxes, laser light head cooling, mobile refrigerators for cars, ice water dispensers, hot and cold compresses, small refrigerators, blood analyzers, and so on. Use the refrigerating end and heating end of the refrigeration chip to cool or heat up the water, and use the current to change the front of the refrigeration chip into a refrigeration or heating end. As long as the direction of the current is changed, the hot and cold ends of the refrigeration chip can be reversed to achieve elevation Temperature function.

The production steps are as follows: (1) Design and draw a simple block diagram; as shown in Figure 2 (2) Arrangement and design of LED strips. (3) Compare the LED strips placed in front, middle, and back of the acrylic. (4) ADC0804 analog to digital conversion. (5) 89C51 programming and testing. (6) The value set by the LCD display. (7) The voltage is adjusted to $5 \mathrm{~V}$ to make the power supply stable output. (8) Use a breadboard to test the circuit. (9) Draw the circuit diagram. (10) Complete the finished product of the topic.

89C51 single-chip program instructions and action descriptions are shown in Figure 3. When the reference voltage measured by the humidity module is sent to ADC0804 for analog-digital conversion, the converted digital signal is judged by 89C51 and sent to the LCD display for display. Therefore, the 89C51 microprocessor sends a signal to the LCD display so that the humidity of the LCD display reaches the set value; And the dehumidification effect of the operation of the cooling chip and the fan is activated. However, when the 89C51 transmits a signal to the LCD display to reduce the humidity value to less than $10 \%$, the operation of the cooling chip and the fan will stop. 


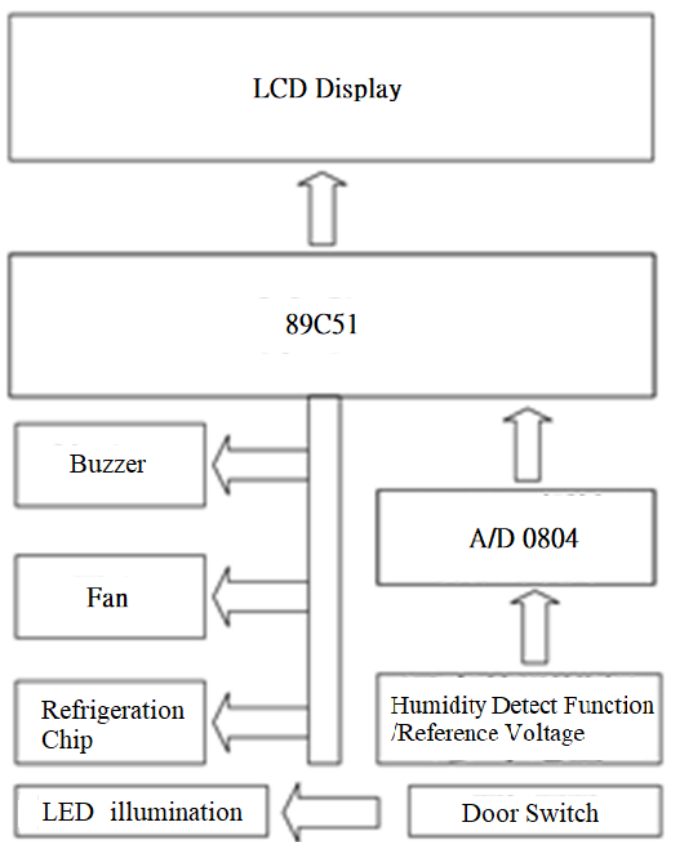

Figure 2. Block diagram of humidity exhaust fan circuit with auxiliary lighting

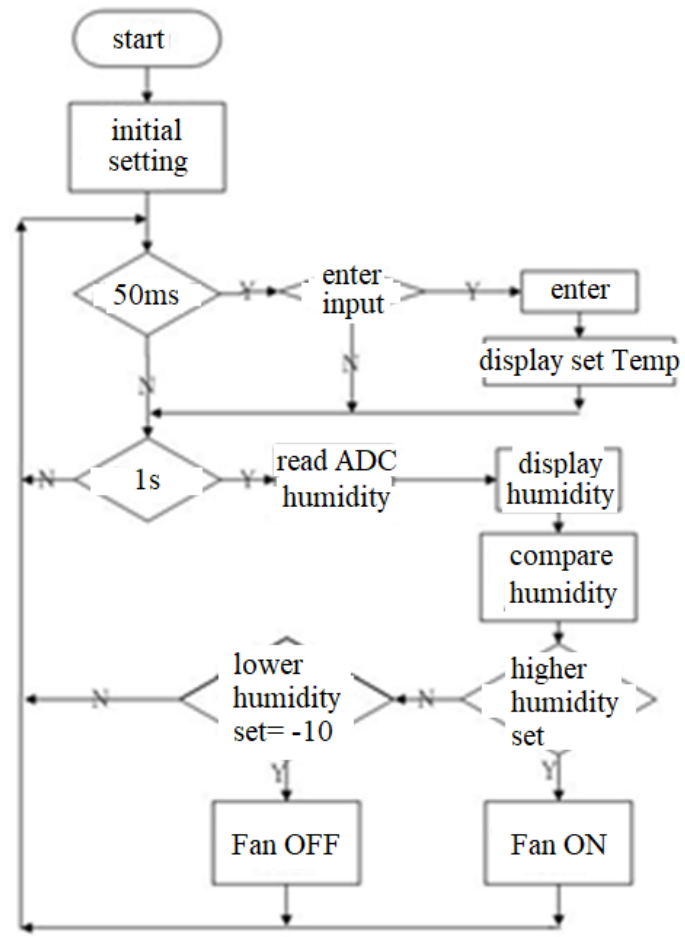

Figure 3. Program flow chart 
The input power is converted into a DC output through a transformer, and the circuit is driven after voltage stabilization. The humidity module senses a certain humidity reference value. When the reference voltage measured by the humidity module is sent to ADC0804 for analog-to-digital conversion, the converted digital signal is judged by 89C51 and sent to the LCD display for display. Therefore, the 89C51 judges that the humidity control reaches the set value, which will activate the cooling chip and The fan starts to dehumidify by cooling, and the dehumidification effect is judged by the digital signal converted by ADC0804 analog to 89C51, then it will be transmitted to the LCD display and dropped to less than $10 \%$ of the set value. The cooling chip and fan operation will stop. The finished photo of the main circuit board is shown in Figure 4.

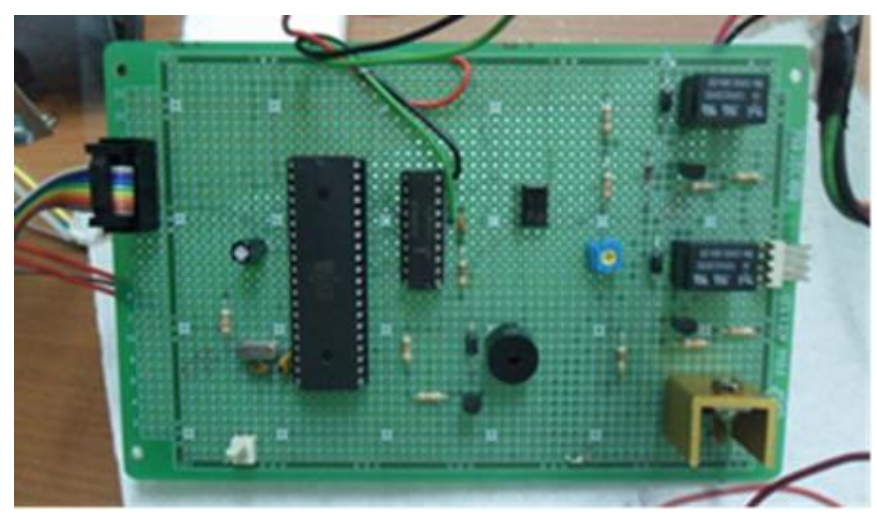

Figure 4. Humidity-controlled exhaust fan main circuit board with auxiliary lighting function

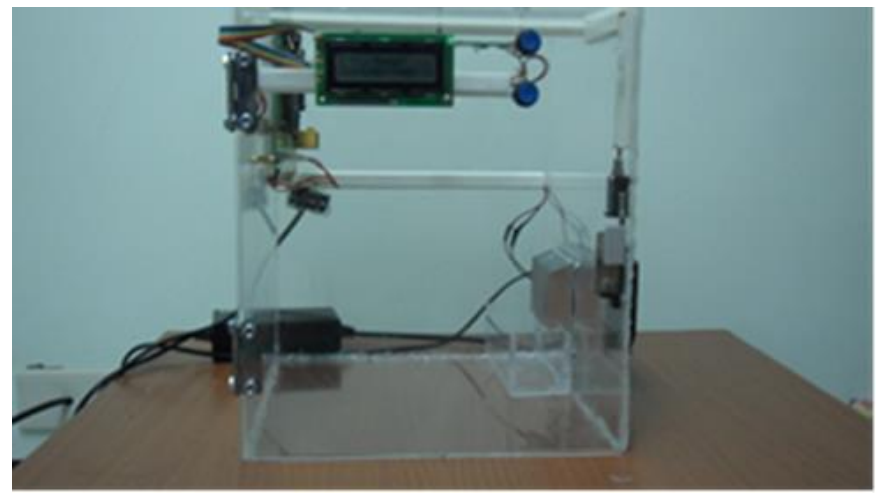

Figure 5. The finished picture of this article

\section{Results}

The finished product of this topic is shown in Figure 5; the value displayed on the LCD display can be controlled by the AT89C51 program, and the humidity value can be controlled; the value displayed after dehumidification. The combined use of a fan and a refrigerating chip makes the dehumidification effect obvious. In addition, the lack of light in the cabinet makes it inconvenient to take things, so the white LED lighting function is added, as shown in Figure 6. Coupled with the application of fans and cooling chips, it can not only achieve energy-saving, power-saving and environmental protection, but also achieves less space and 
convenient device use. The periphery is made of acrylic board and glued, as shown in Figure 6. The thematic door is opened and the white light LED is set.

The lighting function of the lamp allows people to open the cabinet so that the place where things are placed can be clearly seen without having to turn on the household electric light to consume electricity. And the white light LED lamp can achieve low power consumption and save electricity and have better lighting effects. As shown in Figure 7, the dehumidification function is significant. After 85 minutes of use, the humidity is greatly reduced from $80 \%$ to $30 \%$, achieving the dehumidification function.

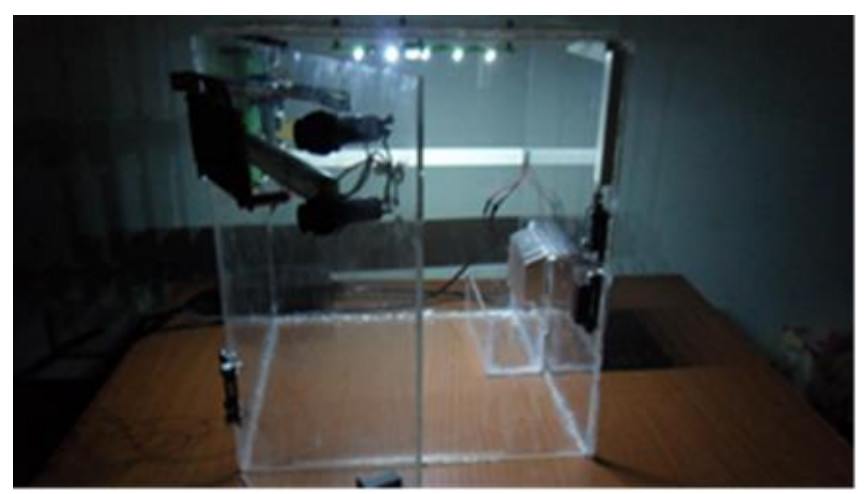

Figure 6. Finished LED lighting picture

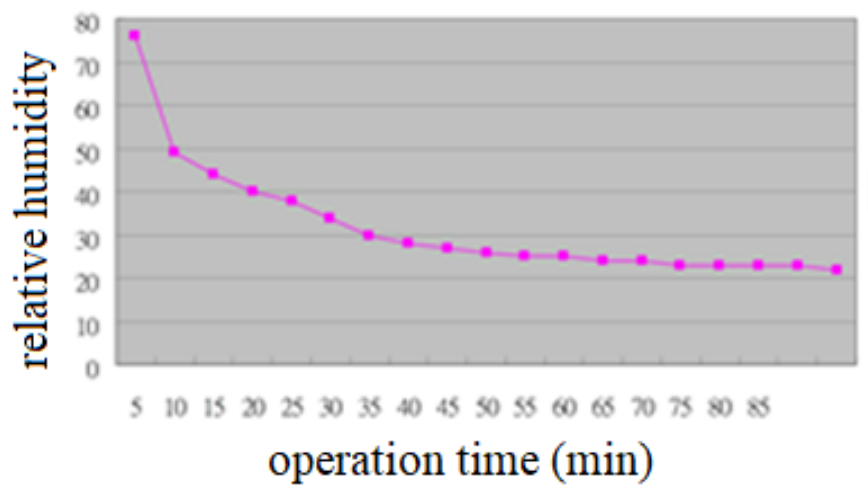

Figure 7. Finished product humidity line chart

\section{Conclusion}

In this work, we use a combination of a fan and a cooling chip, and use humidity sensing control as the sensing dehumidification function and the configuration of white light LED lighting equipment, which not only saves the power consumption of household electric lights, but also has low power consumption for white light LED lighting. It also echoes the reduction in the use of refrigerants to achieve energy saving and green environmental protection. In addition, it also saves space and is convenient for device use. 


\section{Acknowledgements}

Helpful discussion and measurement operation from Prof. W.B. Lin are appreciated during this work.

\section{References}

[1] Bechtold, P., N. Semane, P. Lopez, J.-P. Chaboureau, A. Beljaars, and N. Bormann, J. Atmos. Sci., 71, 734-753 (2014)

[2] Zhang, C. and Y. Wang, J. Climate, 30,5923-5941 (2017)

[3] Nakazawa T., J. Meteor. Soc. Japan, 66, 823-839 (1988)

[4] Liebmann, B., H.H. Hendon, and J.D. Glick, J. Meteor. Soc. Japan, 72, 401-411 (1994)

[5] Chen, G. T. J., and C. Y. Tsay, Tech.Rep. No. Mei-Yu-001, Dept. of Atmos.Sci., Natl. Taiwan Univ., 249 (1977)

[6] Chou, M. D., C. H. Wu, and W. S. Kau, J. Clim., 24, 5081-5093 (2011) 